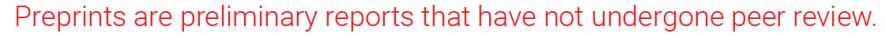 They should not be considered conclusive, used to inform clinical practice, or referenced by the media as validated information. \\ The microbiota diversity following antibiotic treatment in Clostridium difficile infection
}

\section{Dana Binyamin}

Bar ilan university

\section{Orna Nitzan}

Baruch Padeh Medical Center Poriya

\section{Maya Azrad}

Baruch Padeh Medical Center Poriya

\section{Zohar Hamo}

Baruch Padeh Medical Center Poriya

\section{Omry Koren}

bar ilan univrsity

avi peretz ( $\nabla$ aperetz@poria.health.gov.il)

poria mediacl center

\section{Research}

Keywords: Clostridium difficile, Infection, Gut microbiome

Posted Date: March 9th, 2020

DOl: https://doi.org/10.21203/rs.3.rs-16389/v1

License: (c) (i) This work is licensed under a Creative Commons Attribution 4.0 International License.

Read Full License 


\section{Abstract}

Background Clostridium difficile ( $C$. difficile) is a major nosocomial pathogen that infect the human gut and can cause $C$. difficile infection (CDI), a diarrheal disease. A dominant risk factor is antibiotic treatment that disrupts the normal gut microbiota. The aim of the study is to examine the correlation between antibiotic treatment received prior to $C$. difficile infection (CDI) onset and patient gut microbiota

Methods Stool samples were collected from patients with CDI, presenting at the Baruch Padeh Medical Center Poriya, Israel. Demographic and clinical information, including previous antibiotic treatments, was collected from patient charts, and CDI severity score was calculated. Bacteria were isolated from stool samples, and gut microbiome was analyzed by sequencing the 16S rRNA gene using the lllumina MiSeq platform and QIIME2.

Results In total, 84 patients with $C$. difficile infection were enrolled in the study; all had received antibiotics prior to disease onset. Due to comorbidities, 46 patients (55\%) received more than one class of antibiotics. The most common class of antibiotics used was cephalosporins ( $n=44$ cases). The intestinal microbiota of the patients was not uniform. Differences in intestinal microbiome were influenced by the different combinations of antibiotics that the patients received $(p=0.022)$

Conclusions The number of different antibiotics combinations has a major impact on the CDI patients gut microbiome, mainly on bacterial richness.

\section{Introduction}

Clostridium difficile (C. difficile) is a Gram-positive, obligate anaerobic bacterium that is a member of the Firmicutes phylum. Its highly resistant spores survive on surfaces for long periods, rendering it highly transmissible from person to person. This occurs mainly in hospitalization facilities, categorizing $\mathrm{C}$. difficile infection (CDI) as a nosocomial infection. ${ }^{1}$ This bacterium can also colonize the gut asymptomatically, potentially leading to a "silent" onward transmission. ${ }^{2}$ The major risk factor for CDI is antibiotic administration, which triggers diarrheal diseases, termed antibiotic-associated diarrhea. ${ }^{3}$ Although nearly all antimicrobial classes have been associated with CDI, clindamycin, third-generation cephalosporins, fluoroquinolones, and penicillins are most commonly implicated. ${ }^{4}$

The gut microbiome plays an central role in CDI. The human body is colonized by a large number of microorganisms, including bacteria, fungi, parasites, and viruses, together termed the human microbiome, whose composition is influenced by several factors, such as diet and host genetics. ${ }^{5}$ Opportunistic pathogens are primarily blunted by activation of the immune system. ${ }^{6}$ This colonization resistance is altered by antibiotics; bacterial composition, richness, and diversity change (dysbiosis) several days after antibiotic administration, generating a convenient niche for spore germination, proliferation, and toxin production. ${ }^{4}$ Indeed, data from human studies have shown that the presence of C. difficile, either as a colonizer or as a pathogen, is associated with reduced microbiota diversity. The various antibiotics 
families may have differential effects on the gut microbiota, thus varying in their impact on

predisposition for $\mathrm{CDI}$. The current study examined the changes in the gut microbiota occurring following antibiotic treatment of patients with C. difficile infection.

\section{Methods}

\section{Study population}

The study population consisted of adults diagnosed with CDI at the Baruch Padeh Medical Center Poriya, Israel. Patients signed a consent form or had a legal guardian sign in their place. Pregnant women and patients suffering from mental illness were not eligible to participate in the study. The study was approved by the Helsinki Committee of the Medical Center, approval No. 0003-15 POR.

\section{Sample collection}

C. difficile identification in stool samples was performed at the Clinical Microbiology Laboratory, as part of the routine $\mathrm{CDI}$ screening procedures. A polymerase chain reaction (PCR) test was performed using the GeneXpert C. difficile PCR assay (Cepheid, Sunnyvale, CA, USA), to identify toxin B, binary toxin, and tcdC deletion.

\section{Severity score calculation}

To classify CDI severity, the severity score index (SSI) was calculated according to the "Score indices for Clostridium difficile infection severity", ${ }^{7}$ which incorporates nine parameters that are associated with increased CDI morbidity and mortality. One point is given for each of the following parameters: altered mental status, abdominal pain or distention, $1500>$ white blood cell $(\mathrm{WBC})>20,000$ cells per cubic meter, hypoalbuminemia ( $<2.5 \mathrm{mg} / \mathrm{dL}$ albumin, ALB), ascites or colitis (imaging), mean arterial pressure (MAP) $<65 \mathrm{mmHg}$, tachycardia $\geq 110$ beats/min, intensive care unit (ICU) transfer. A score of $0-3$ reflects mild disease, 4- 6 moderate disease, and $\geq 7$ severe disease.

The following demographic and clinical data were collected from the medical records: age, gender, community- versus hospital-acquired CDI, death during hospitalization, and laboratory test (leukocyte count, serum albumin, C-reactive protein (CRP)) results.

\section{Gut microbiome}

DNA extraction, amplification and sequencing: DNA was extracted from $0.25 \mathrm{ml}$ liquid fecal samples using the Power Soil DNA Isolation Kit (MoBio, Carlsbad, USA), according to manufacturer's instructions and following a 2-min bead-beating step (Biospec). ${ }^{8}$ DNA was stored at $-20{ }^{\circ} \mathrm{C}$ until use.

From the extracted DNA, the V4 region of the bacterial 16S rRNA gene was amplified using the $515 \mathrm{~F}$ and 806R barcoded primers, as per the Earth Microbiome Project protocol. ${ }^{9}$ PCR reaction conditions included an initial denaturing step for 3 min at $95^{\circ} \mathrm{C}$, followed by 30 cycles of denaturation ( $98^{\circ} \mathrm{C}$ for $10 \mathrm{sec}$ ), annealing $\left(55^{\circ} \mathrm{C}\right.$ for $\left.5 \mathrm{sec}\right)$, and extension $\left(72^{\circ} \mathrm{C}\right.$ for $\left.20 \mathrm{sec}\right)$, with a final elongation at $72^{\circ} \mathrm{C}$ (for $\left.1 \mathrm{~min}\right)$. 
PCR products were purified using AMPure magnetic beads (Beckman Coulter, Florida, USA), ${ }^{10}$ and quantified using the Qubit dsDNA HS Assay (Thermo Fisher, Bartlesville, USA). ${ }^{11}$ Samples were then pooled to equal concentrations (50 $\mathrm{ng} / \mu \mathrm{l})$ and purified again using $2 \% \mathrm{E}-\mathrm{Gel}$ agarose inserted in an E-Gel PowerBase device (Invitrogen, Carlsbad, USA). Then, DNA fragments were purified from the agarose using NucleoSpin ${ }^{\circledR}$ Gel and PCR Clean-up kit (Macherey-Nagel, Düren, Germany), and sequenced using the Illumina MiSeq platform at the Genomic Center, Faculty of Medicine, Bar-llan University, Israel.

Analysis: Data analysis was performed using QIIME2. ${ }^{12}$ Sequence reads were demultiplexed by persample barcodes and Illumina-sequenced amplicon read errors were corrected by the Divisive Amplicon Denoising Algorithm (DADA2) ${ }^{13}$. A phylogenetic tree was generated and taxonomy was classified using Greengenes reference database at a confidence threshold of $99 \% .{ }^{14}$ Alpha and beta diversities were calculated based on a feature table containing features observed in at least 40 samples (50\%) and on samples containing at least 8000 sequences. Richness was calculated using Faith's Phylogenetic Diversity, a qualitative measure of community richness that incorporates phylogenetic relationships between taxa. ${ }^{15}$ Beta diversity was analyzed using Principal Coordinate Analysis (PCoA) based on weighted UniFrac distance matrices. ${ }^{16}$

\section{Statistical Analysis}

In Faith's phylogenetic diversity measure, differences between groups were analyzed by the Kruskal-Wallis (pairwise) test. Differences between weighted UniFrac distances were analyzed by pairwise Permanova test. Statistical significance was defined as $p<0.05$.

\section{Results}

\section{Demographic and clinical profiles}

Overall, 84 CDI patients, of an average age of $72.42 \pm 16.74$ years, were enrolled in this study (Table 1). Of the 84 patients, 41 (48.81\%) were women. Seventeen patients $(20.24 \%)$ died during hospitalization. More than half of the patients (55.95\%) had a nosocomial CDI. Disease severity was calculated for 81 patients, as medical information of three ICU patients was inaccessible; 58 (71.6\%) patients suffered from mild disease, 20 (24.7\%) from moderate disease, and $3(3.7 \%)$ from severe disease. All patients received antibiotics prior to disease onset due to other illnesses; 38 patients $(45.24 \%)$ received one class of antibiotics, 32 patients (38.1\%) received two classes, 10 patients $(11.9 \%)$ received three classes, and 4 patients $(4.76 \%)$ received 4 classes.

Table 1: Demographic and baseline characteristics of $C$. difficile infection patients $(\mathrm{N}=84)$ 


\begin{tabular}{|c|c|}
\hline \multicolumn{2}{|l|}{ Parameter } \\
\hline Age (years), mean \pm SD & $72.42 \pm 16.74$ \\
\hline \multicolumn{2}{|l|}{ Gender, n (\%) } \\
\hline Male & $41(48.81)$ \\
\hline Female & $43(51.19)$ \\
\hline \multicolumn{2}{|l|}{ Infection acquisition, $\mathrm{n}(\%)$} \\
\hline Nosocomial & $47(55.95)$ \\
\hline Community-acquired & $37(44.05)$ \\
\hline In-hospital mortality, n (\%) & $67(79.76)$ \\
\hline Alive & $17(20.24)$ \\
\hline \multicolumn{2}{|l|}{ Dead during hospitalization } \\
\hline \multicolumn{2}{|l|}{ Disease Severity*, n(\%) } \\
\hline Mild & $58(71.6)$ \\
\hline Moderate & $20(24.7)$ \\
\hline Severe & $3(3.7)$ \\
\hline \multicolumn{2}{|c|}{ Number of antibiotic classes received before CDI onset, $n(\%)$} \\
\hline \multicolumn{2}{|l|}{1} \\
\hline 2 & $38(45.24)$ \\
\hline 3 & $32(38.1)$ \\
\hline \multirow[t]{2}{*}{4} & $10(11.9)$ \\
\hline & $4(4.76)$ \\
\hline
\end{tabular}

* Disease severity was calculated for 81 patients only.

\section{Antibiotics received before CDI onset}

Twelve classes of antibiotics taken before CDI onset were recorded (Table 2). Antibiotics from the cephalosporin class were most widely used $(n=44)$, followed by penicillin $(n=32)$, which was often administered with other antibiotics $(n=24)$. Sulfa antibiotics were used in only 2 patients and tetracyclines in one patient. In 18 cases, one antibiotic from the cephalosporin class was sufficient to 
trigger disease, but CDI was often diagnosed after treatment with cephalosporin in combination with other antibiotics $(n=26)$.

Table 2

Antibiotics classes received by CDI patients (Some patients received more than one antibiotic)

\begin{tabular}{|llllll|}
\hline Antibiotic Class & $\begin{array}{l}\mathbf{1} \text { class of } \\
\text { antibiotic } \\
\mathbf{n = 3 8}\end{array}$ & $\begin{array}{l}\mathbf{2} \text { classes of } \\
\text { antibiotic } \\
\mathbf{n = 3 2}\end{array}$ & $\begin{array}{l}\mathbf{3} \text { classes of } \\
\text { antibiotic } \\
\mathbf{n}=\mathbf{1 0}\end{array}$ & $\begin{array}{l}\mathbf{4} \text { classes of } \\
\text { antibiotic } \\
\mathbf{n}=\mathbf{4}\end{array}$ & Overall \\
\hline Aminoglycoside & 2 & 6 & 0 & 3 & 11 \\
\hline Carbapenem & 2 & 4 & 3 & 0 & 9 \\
\hline Cephalosporin & 18 & 17 & 7 & 2 & 44 \\
\hline Chloramphenicol & 2 & 3 & 0 & 1 & 6 \\
\hline Glycopeptide & 0 & 2 & 4 & 2 & 8 \\
\hline Lincosamides & 4 & 1 & 1 & 1 & 7 \\
\hline Macrolide & 1 & 1 & 4 & 0 & 6 \\
\hline Penicillin & 8 & 16 & 6 & 2 & 32 \\
\hline Quinolone & 1 & 6 & 2 & 2 & 11 \\
\hline Tetracycline & 0 & 1 & 0 & 1 & 2 \\
\hline Trim/sulfa & 0 & 1 & 0 & 0 & 11 \\
\hline Other & 0 & 6 & 3 & 2 & 1 \\
\hline
\end{tabular}

\section{Microbiome Analysis}

Microbiome analysis was performed on 67 out of 84 CDI patient samples (79.76\%); 17 samples (20.24\%) did not pass quality control. Proteobacteria were the dominant phylum in $50 \%$ of samples, followed by Bacteroidetes (20\%) (Fig. 1) and Firmicutes and Verrucomicrobia phyla (12\%) (Fig. 1A). At the family level, Enterobacteriaceae and Bacteroidaceae each dominated $20 \%$ of the patient samples and Verrucomicrobiaceae dominated $11 \%$ of the samples. Other families were present in smaller percentages (Fig. 1B).

Calculation of beta-diversity in CDI patients who received different combinations of antibiotics (Fig. 2A) showed that only patients who had received four classes of antibiotics clustered significantly distant from the other groups $(p<0.05)$. Bacterial richness (alpha-diversity) negatively correlated with the number of antibiotics received (Fig. 2B). Bacterial richness among CDI patients who had received four classes of 
antibiotics was the lowest and was significantly different from the other groups $\left(\left({ }^{\star} p=0.03,{ }^{*}{ }^{1} p=0.007\right.\right.$, $\star * 2 \mathrm{p}=0.005)$.).

\section{Discussion}

The current study examined the correlation between gut bacterial composition of CDI patients and antibiotic treatment received prior to infection onset. The epidemiological data of the study population was in correlation with the known characteristics of CDI patients, i.e., older age and high mortality rate. Additionally, the numbers of nosocomial- and community-acquired cases were similar to earlier reports ${ }^{17}$. Recently, the prevalence of community-acquired infections has increased due to elevated use of antibiotics that were previously only administered in hospitals via intravenous infusion. ${ }^{17}$

Most patients were diagnosed with mild disease, while only a few were diagnosed with severe disease. These results point to an increase in the prevalence of moderate-severe CDI compared to a study conducted in 2016 in northern Israel which found that most patients had mild disease, a few had moderate disease, and none were diagnosed with a severe disease. ${ }^{18}$ This increase in disease severity can be attributed to an increase in antibiotic resistance or emergence of more virulent strains. ${ }^{1}$

The majority of patients received one or two classes of antibiotics prior to CDI onset, corresponding with previous reports demonstrating that one type of antibiotic is sufficient to induce CDI. ${ }^{4}$ Cephalosporins and penicillins were the most commonly used antibiotics, two drugs which have previously been shown to significantly increase the risk of $\mathrm{CDI}$ as compared to other antibiotics. ${ }^{4,19-20}$ Fluoroquinolone and clindamycin have also been highly correlated with CDI development, yet, in our study, only a small percentage of patients received these antibiotics.

Examination of intestinal bacterial populations of CDI patients and their correlation with previous antibiotic treatment, showed that there was no phylum and family level composition common to all CDI patients, as has been described in other studies. ${ }^{21-23}$ In their study comparing the gut microbiome profile of $C D I$ versus non-CDI patients, Manges et al. found an increase in Firmicutes, Proteobacteria and Actinobacteria phyla, as well as a decrease in Bacteroidetes. ${ }^{24}$ Antharam et al., surveying the distal gut microbiota of individuals with CDI, found that these patients had significantly less diverse communities, particularly a less diverse Firmicutes population than patients with non-CD diarrhea or healthy controls. ${ }^{21}$ In addition, there was depletion of gut commensals such as the Ruminococcaceae and Lachnospiraceae families and butyrate-producing anaerobic fermenters. This lack of uniformity can be explained by the various factors affecting the intestinal bacteria, such as nutrition, ${ }^{25}$ although we tried to control for these factors during data analysis. More specifically, several parameters (such as age, gender, and disease severity) were tested; none of them had significant effects on bacterial population. In contrast, we found that the antibiotic combination administered to CDI patients before disease onset correlated with the intestinal microbiota. Patients who had received four classes of antibiotics had more similar microbiomes. In addition, and inverse correlation between bacterial richness and the number of 
antibiotics received was noted, with significant differences between patients who received four classes of antibiotics versus those who received 1 or 2 classes of antibiotics. These findings can likely be ascribed to the broader range of bacterial species targeted by multi-class antibiotic treatment regimens, which subsequently leads to reduced microbiota richness. A limitation of this study is lack of a comparison to gut microbiome of healthy condition due to the difficulty in finding healthy elder controls. Such a comparison could have allowed to understand the importance of the gut microbiota's capability of providing colonization resistance against $\mathrm{C}$. difficile.

\section{Conclusions}

In conclusion, no uniform microbiome profile was observed among the tested CDI patients. Antibiotics are the main cause for disease onset and indeed we saw that all CDI patients received antibiotics. However, the major influence on the gut microbiome is in patient who received 4 different antibiotics classes, which demonstrated significantly lower richness and diversity compare to patients who received less then 4 different antibiotics classes.

\section{Abbreviations}

ALB: albumin; CDI: C. difficile infection; CRP: C-reactive protein; ICU: intensive care unit; MAP: mean arterial pressure; PCoA: Principal Coordinate Analysis; PCR: polymerase chain reaction; SSI: severity score index; WBC: white blood cell

\section{Declarations}

Ethics approval and consent to participate: The study was approved by the Helsinki Committee of the Medical Center, committee approval number- 0003-15 POR.

Consent for publication:

Not applicable

Availability of data and materials:

The datasets used and/or analysed during the current study are available from the corresponding author on reasonable request.

Competing interests: The authors declare that they have no competing interests.

Funding: This research did not receive any specific grant from funding agencies in the public, commercial, or not-for-profit sectors

Authors' contributions: DN, MA, OK and AP designed the study, analyzed, interpreted the data, and wrote the final manuscript. $\mathrm{ZH}$ and $\mathrm{ON}$ were involved in development of the protocols and contributed to the 
biobank sample or data collection. All authors read and approved the final manuscript.

Acknowledgements: Not applicable

\section{References}

1. Xia J, Gao J, Tang W. Nosocomial infection and its molecular mechanisms of antibiotic resistance. Biosci 2016;10:14-21.

2. Clabots $\mathrm{CR}$, Johnson $\mathrm{S}$, Olson $\mathrm{MM}$ et al. Acquisition of Clostridium difficile by hospitalized patients: evidence for colonized new admissions as a source of infection. J Infect Dis. 1992;166:561-7.

3. Högenauer C, Hammer HF, Krejs GJ, Reisinger EC. Mechanisms and management of antibioticassociated diarrhea. Clin Infect Dis. 1998;27:702-10.

4. Owens Jr RC, Donskey CJ, Gaynes RP et al. Antimicrobial-associated risk factors for Clostridium difficile infection. Clin Infect Dis. 2008;46(S1):S19-31.

5. Human Microbiome Project Consortium. Structure, function and diversity of the healthy human microbiome. Nature. 2012;486:207-14.

6. Monaghan TM. New perspectives in Clostridium difficile disease pathogenesis. Infect Dis Clin. 2015;29:1-11.

7. Velazquez-Gomez I, Rocha-Rodriguez R, Toro DH et al. A severity score index for Clostridium difficile infection. Infect Dis Clin Pract. 2008;16:376-8.

8. Santos SS, Nielsen TK, Hansen LH et al. Comparison of three DNA extraction methods for recovery of soil protist DNA. J Microbiol Methods. 2015;115:13-9.

9. Wu L, Wen C, Qin Y et al. Phasing amplicon sequencing on Illumina Miseq for robust environmental microbial community analysis. BMC Microbiol. 2015;15:125.

10. Meyer M, Kircher M. Illumina sequencing library preparation for highly multiplexed target capture and sequencing. Cold Spring Harb Protoc. 2010;2010:pdb-prot5448.

11. Nakayama $\mathrm{Y}$, Yamaguchi $\mathrm{H}$, Einaga $\mathrm{N}$ et al. Pitfalls of DNA quantification using DNA-binding fluorescent dyes and suggested solutions. PLoS One. 2016;1:e0150528.

12. Caporaso JG, Kuczynski J, Stombaugh J et al. QIIME allows analysis of high-throughput community sequencing data. Nat Methods. 2010;7:335-6.

13. Callahan BJ, McMurdie PJ, Rosen MJ et al. DADA2: high-resolution sample inference from Illumina amplicon data. Nat Methods. 2016;13:581-3.

14. DeSantis TZ, Hugenholtz P, Larsen N et al. Greengenes, a chimera-checked 16S rRNA gene database and workbench compatible with ARB. Appl Environ Microbiol. 2006;72:5069-72.

15. Faith DP. Conservation evaluation and phylogenetic diversity. Biol Conserv. 1992;61:1-10.

16. Lozupone C, Knight R. UniFrac: a new phylogenetic method for comparing microbial communities. Appl Environ Microbiol. 2005;71:8228-35. 
17. Warriner $\mathrm{K}, \mathrm{Xu} \mathrm{C}$, Habash $\mathrm{M}$, et al. Dissemination of Clostridium difficile in food and the environment: Significant sources of difficile community-acquired infection? J Appl Microbiol. 2017;122:542-53.

18. Peretz A, Tkhawkho L, Pastukh $\mathrm{N}$ et al. Correlation between fecal calprotectin levels, disease severity and the hypervirulent ribotype 027 strain in patients with Clostridium difficile BMC Infect Dis. 2016;16:309.

19. Debast SB, Vaessen N, Choudry A et al. Successful combat of an outbreak due to Clostridium difficile PCR ribotype 027 and recognition of specific risk factors. Clin Microbiol Infect. 2009;15:427-34.

20. Goudarzi M, Seyedjavadi SS, Goudarzi H et al. Clostridium difficile infection: epidemiology, pathogenesis, risk factors, and therapeutic options. Scientifica (Cairo). 2014;2014:916826.

21. Antharam VC, Li EC, Ishmael A et al. Intestinal dysbiosis and depletion of butyrogenic bacteria in Clostridium difficile infection and nosocomial diarrhea. J Clin Microbiol. 2013;51:2884-92.

22. Pakpour S, Bhanvadia A, Zhu R et al. Identifying predictive features of Clostridium difficile infection recurrence before, during, and after primary antibiotic treatment. Microbiome. 2017; 5:148.

23. Schneider $D$, Thürmer A, Gollnow K et al. Gut bacterial communities of diarrheic patients with indications of Clostridioides difficile Sci Data. 2017;4:170152.

24. Manges AR, Labbe A, Loo VG et al. Comparative metagenomic study of alterations to the intestinal microbiota and risk of nosocomial Clostridum difficile-associated disease. Infect Dis. 2010;202:187784.

\section{Figures}



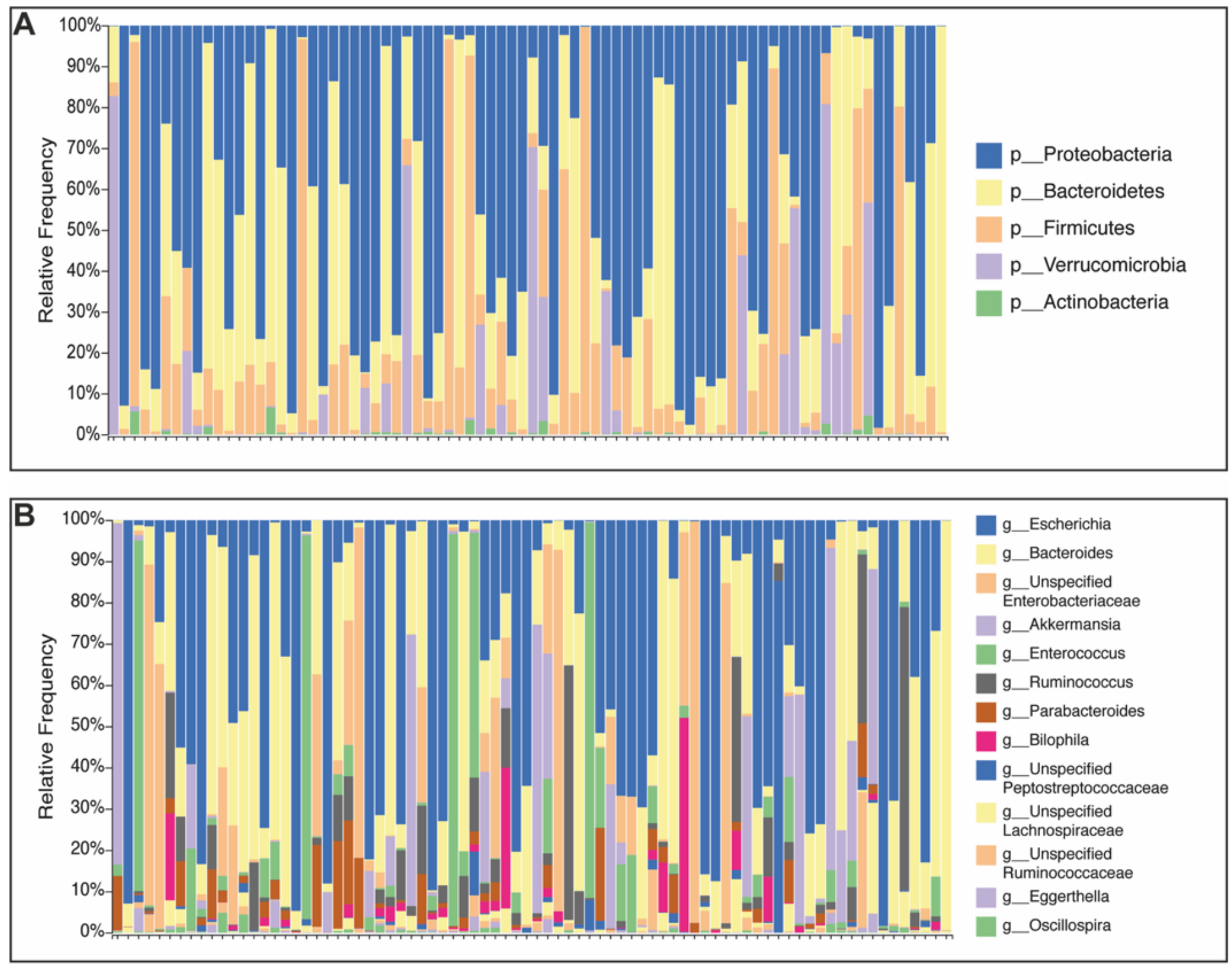

Figure 1

Microbial community structure of $\mathrm{C}$. difficile infection (CDI) patients. The gut microbiota composition of 67 CDI patients. Taxonomy plot at (A) phylum and (B) family levels. 


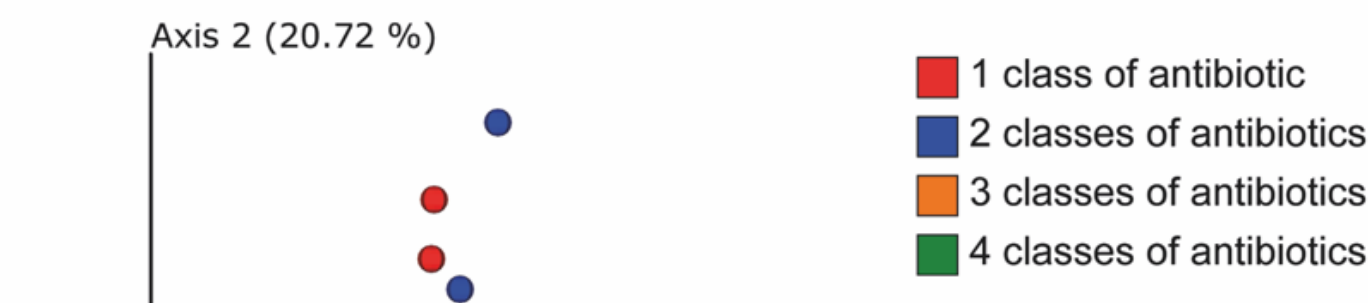

Axis 3 (12.06\%)

B

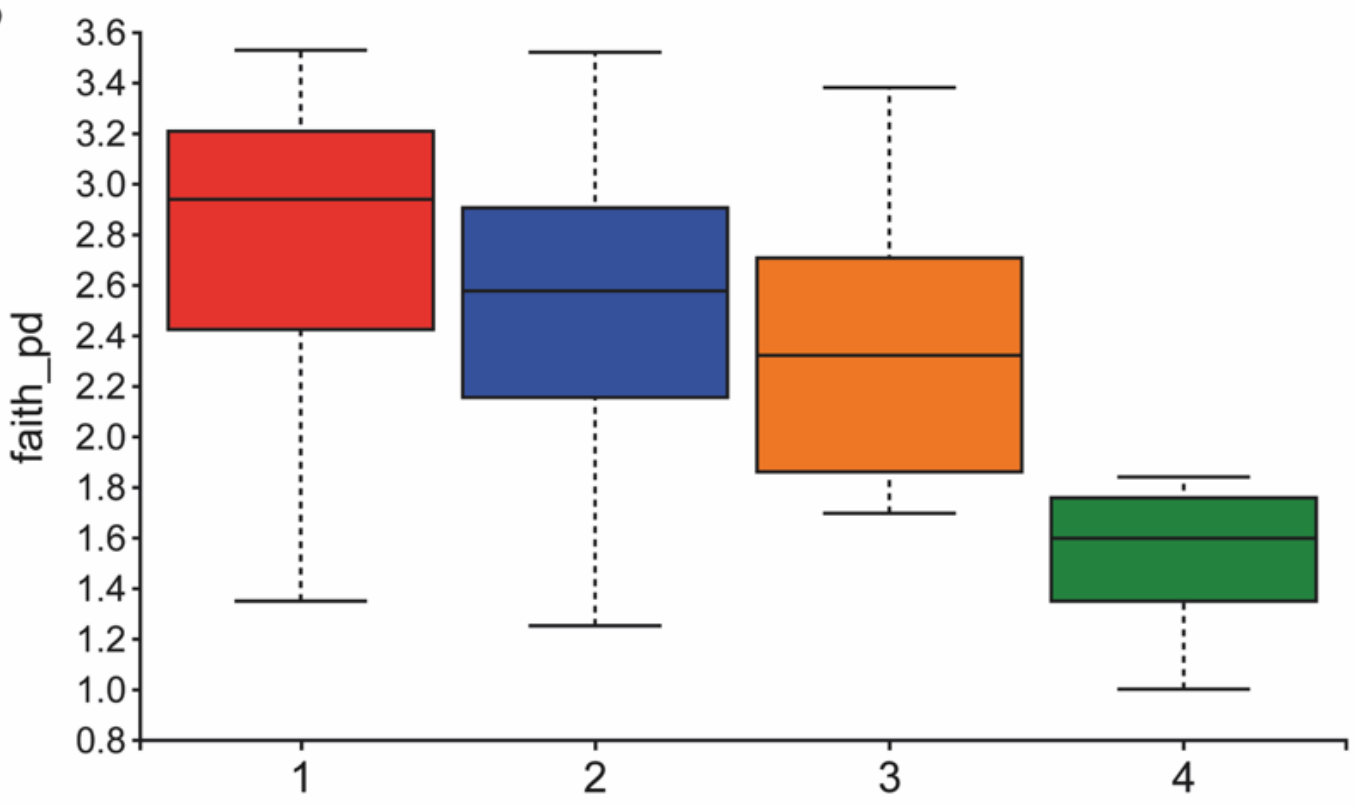

Number of different received antibiotics classes

\section{Figure 2}

Microbial community diversity between and within C. difficile infection (CDI) patients, by combinations of antibiotics received prior to $C D I$ onset. CDI patients were divided to 4 groups according to the number of classes of antibiotics received prior to onset of infection: $1(n=30), 2(n=24), 3(n=9)$, and $4(n=4)$ classes of antibiotics. (A) Beta diversity using weighted UniFrac, (B) Alpha diversity using Faith's Phylogenetic Diversity $\left({ }^{\star} p=0.022\right)$. 